ACCEPTED By THE AStRonOMiCAL JOURNAL

Preprint typeset using LTTE style emulateapj v. 05/04/06

\title{
A SEARCH FOR RAPIDLY SPINNING PULSARS AND FAST TRANSIENTS IN UNIDENTIFIED RADIO SOURCES WITH THE NRAO 43-METER TELESCOPE
}

\author{
Deborah Schmidt ${ }^{1,2,3}$, Fronefield Crawford $^{1}$, Glen LANGSTON $^{2}, \&$ Claire Gilpin $^{1}$, \\ Draft version August 6, 2021
}

\begin{abstract}
We have searched 75 unidentified radio sources selected from the NRAO VLA Sky Survey (NVSS) catalog for the presence of rapidly spinning pulsars and short, dispersed radio bursts. The sources are radio bright, have no identifications or optical source coincidences, are more than 5\% linearly polarized, and are spatially unresolved in the catalog. If these sources are fast-spinning pulsars (e.g. sub-millisecond pulsars), previous large-scale pulsar surveys may have missed detection due to instrumental and computational limitations, eclipsing effects, or diffractive scintillation. The discovery of a sub-millisecond pulsar would significantly constrain the neutron star equation of state and would have implications for models predicting a rapid slowdown of highly recycled X-ray pulsars to millisecond periods from, e.g., accretion disk decoupling. These same sources were previously searched unsuccessfully for pulsations at $610 \mathrm{MHz}$ with the Lovell Telescope at Jodrell Bank. This new search was conducted at a different epoch with a new $800 \mathrm{MHz}$ backend on the NRAO 43-meter Telescope at a center frequency of $1200 \mathrm{MHz}$. Our search was sensitive to sub-millisecond pulsars in highly accelerated binary systems and to short transient pulses. No periodic or transient signals were detected from any of the target sources. We conclude that diffractive scintillation, dispersive smearing, and binary acceleration are unlikely to have prevented detection of the large majority of the sources if they are pulsars, though we cannot rule out eclipsing, nulling or intermittent emission, or radio interference as possible factors for some non-detections. Other (speculative) possibilities for what these sources might be include radio-emitting magnetic cataclysmic variables or older pulsars with aligned magnetic and spin axes.
\end{abstract}

Subject headings: pulsars: general - surveys

\section{INTRODUCTION}

Radio pulsars have a wide range of spin periods, but no pulsar has yet been observed to be rotating faster than PSR J1748-2446ad, which has a spin period of $P=1.39 \mathrm{~ms}$ (Hessels et al. 2006). Models of the neutron star equation of state (EOS) and the properties of pulsars that have been spun up (recycled) via accretion from a companion suggest that pulsars with spin periods less than a millisecond ("submillisecond" pulsars) can exist structurally and could in principle be produced from the recycling process (e.g., Cooke et al. 1994; Haensel et al. 1999). A significant population of sub-millisecond pulsars may therefore be present in the Galaxy waiting to be discovered (see, e.g., Possenti et al. 1998). However, some studies have suggested that a spin equilibrium is reached during accretion which limits the neutron star spin to larger (millisecond) periods. In these scenarios, the spin up from accretion is balanced by angular momentum loss through, e.g., an accretion disk/magnetosphere interaction (Patruno et al. 2012) or the emission of gravitational radiation (Wagoner 1984; Bildsten 1998). Ho et al. (2011) provide a brief overview. A more recent model presented by Tauris (2012) suggests that highly recycled neutron stars can be produced as sub-millisecond X-ray sources during the accretion phase, but that they are then rapidly spun down in the Roche lobe decoupling phase. In this case, the magnetosphere expands during the last phase of accretion, a large fraction of the rotational energy is dissipated, and the pulsar is not seen as a sub-millisecond pulsar when the radio emission turns on.

\footnotetext{
${ }^{1}$ Department of Physics and Astronomy, Franklin and Marshall College P.O. Box 3003, Lancaster, PA 17604, USA

${ }^{2}$ National Radio Astronomy Observatory, P.O. Box 2, Green Bank WV 24944

${ }^{3}$ Department of Astronomy, University of Arizona, 933 North Cherry Avenue, Tucson, AZ 85721, USA
}

The discovery of a sub-millisecond pulsar would be of particular importance since it would falsify models suggesting that sub-millisecond radio pulsars should not be observed (see above) while also placing significant constraints on the largely unknown EOS of neutron star matter (e.g., Lattimer \& Prakash 2004). However, sub-millisecond pulsars are difficult to detect owing to a number of selection effects. These include limitations in observing sensitivity, digital sampling rates, and frequency channel resolution, as well as eclipsing and acceleration effects if the pulsar is in a tight binary system. In fact, very rapidly spinning pulsars may be preferentially located in such eclipsing systems (Hessels et al. 2006; Hessels 2008), making such systems even harder to detect. Large-area surveys having the necessary time and frequency resolution (as well as the required post-observation computational power for data processing) to detect such pulsars have not yet been feasible on the full sky. Therefore, targeting smaller areas likely to harbor pulsars remains one of the most promising avenues for trying to discover a sub-millisecond pulsar. Examples of this approach include the highly successful deep targeted radio searches of Fermi $\gamma$-ray sources (Ray et al. 2012, 2013) in which an astonishing 44 new millisecond pulsars (MSPs) have recently been discovered.

Some pulsars are also known to exhibit detectable single pulses at irregular intervals. Notable examples of this type include the Crab pulsar (Staelin \& Reifenstein 1968) and the MSP PSR B1937+21 (Cognard et al. 1996), both of which are also detectable as periodic sources. Other, more recently discovered sources of sporadic radio emission include rotating radio transients (RRATs; McLaughlin et al. 2006), which are rotating neutron stars that are only detectable as transient burst sources.

In an effort to discover previously undetected millisecond and sub-millisecond pulsars, as well as to find new transient 
emitters, we have searched a sample of unidentified radio sources from the NRAO VLA Sky Survey (NVSS) (Condon et al. 1998). These sources were originally selected by Crawford et al. (2000) for a similar pulsar search with the 76-m Lovell Telescope at the Jodrell Bank Observatory. We outline the source selection criteria below, which can also be found in Crawford et al. (2000). The NVSS is a large-scale radio survey of the northern sky at $1400 \mathrm{MHz}$ conducted with the Very Large Array (VLA). It covered declinations $\delta>-40^{\circ}$ in $\mathrm{D}$ and DnC configurations with a synthesized beam size of $45^{\prime \prime}$. Position errors in the NVSS catalog are typically $\lesssim 1^{\prime \prime}$ for strong sources. The catalog contains $\sim 3 \times 10^{5}$ sources with a flux density greater than $15 \mathrm{mJy}$, when local sources and unreliable survey regions are excluded (see, e.g., Blake \& Wall 2002; Crawford 2009). Many of the sources detected in the NVSS remain unidentified.

Owing to the large number of extended, resolved sources in the catalog, it was necessary to explicitly check for the pointlike nature of those objects. Sources were checked for corresponding detections in the Faint Images of the Radio Sky at Twenty Centimeters (FIRST) survey (Becker et al. 1995). The FIRST survey covered the north and south Galactic caps using the VLA in B configuration with a synthesized beam size of $5^{\prime \prime} .4$. For sources found in both the NVSS and FIRST catalogs, the corresponding flux densities were compared. If a source was extended, the better resolution of the FIRST survey would result in a lower flux density than the NVSS survey, since some of the flux would be resolved out. Therefore, sources were only included if their FIRST and NVSS flux densities agreed to within a few percent (indicating an unresolved, non-variable source) or if the FIRST flux density exceeded the NVSS flux density (indicating an unresolved scintillating source). For those sources outside of the FIRST survey region, observations were performed using the VLA in $\mathrm{B}$ configuration to achieve the same angular resolution as the FIRST survey. The flux densities of the sources observed in these pointings were then compared with their NVSS values to eliminate any additional extended sources.

The target list was further reduced by requiring that the sources have no identifications with known sources (and no optical source coincidences), be radio bright (with a 1400 MHz flux density $\geq 15 \mathrm{mJy}$ ), be spatially unresolved in the NVSS catalog, and be more than $5 \%$ linearly polarized. The polarization criterion was used since pulsars often have a high degree of linear polarization (Lyne \& Manchester 1988), which makes polarized point radio sources good candidates to search. Although there is not a clear polarization cutoff separating the pulsar and extragalactic populations, a polarization threshold of $5 \%$ excludes about $90 \%$ of the identified nonpulsar population while retaining about $90 \%$ of the identified pulsar population (Han \& Tian 1999; Crawford et al. 2000).

Using these criteria, a list of 92 target sources was compiled and searched by Crawford et al. (2000) for radio pulsations using the 76-m Lovell Telescope. No pulsations were detected from any of the target sources in that search, but no search for single pulses was done at that time. A recent re-analysis of these data using a search for single pulses revealed no new detections. Since the completion of the Crawford et al. (2000) survey, 16 of the 92 target sources have been identified as extragalactic objects in SIMBAD 4 (see Table 1). Some of the targets have also been detected in other radio surveys (listed in Table 1) at different frequencies. In these cases we measured

\footnotetext{
${ }^{4}$ http://simbad.u-strabg.fr/simbad
}

spectral indices for them (see Table 1). All of the measured spectral index values are shallower than the average pulsar spectral index of $\alpha=-1.6$ (Lorimer et al. 1995), where $\alpha$ is defined according to $S \sim \nu^{\alpha}$ ( $S$ is flux density and $\nu$ is the observing frequency). This does not preclude these sources being pulsars, but they are not as steep as expected.

For our search, we have used the same set of selection criteria but have excluded the 16 sources that now have secure extragalactic identifications. One remaining source was not observed owing to telescope scheduling limitations, leaving 75 unidentified targets that we observed and searched. We checked the Fermi LAT 2-year Point Source Catalog (2FGL) (Nolan et al. 2012) for coincidences with our targets and found that none lie within the $95 \%$ error ellipses of any of the Fermi sources. Table 1 presents the full list of 92 sources from Table 1 of Crawford et al. (2000). The 16 secure extragalactic identifications are indicated, as are the measured radio spectral index values (where available). This list represents just a small fraction $(<0.1 \%)$ of the total number of sources in the NVSS catalog with flux densities greater than $15 \mathrm{mJy}$. Note that the flux densities and polarization fractions listed were obtained from revision 2.18 of the NVSS catalog 5 and in some cases are slightly different from the earlier catalog values presented in Table 1 of Crawford et al. (2000).

\section{MOTIVATION FOR NEW OBSERVATIONS}

Starting in February of 2010, we conducted new observations of the 75 unidentified sources with the NRAO 43meter Telescope ${ }^{6}$ at the Green Bank Observatory. Several factors motivated this. The first was the availability of the PRESTO $^{7}$ and SIGPROC ${ }^{8}$ software packages, which are now widely used for pulsar data analysis (Ransom 2001; Ransom et al. 2002). PRESTO and the single pulse search capability of SIGPROC were not available at the time of the search of Crawford et al. (2000). The development of the Fourierbased search code in PRESTO for acceleration searches was also a major advancement for detecting binary pulsar systems in our search. The ability to conduct acceleration searches of the survey data is especially important for the detection of millisecond and sub-millisecond pulsars since approximately $80 \%$ of MSPs are known to be in binaries (e.g., Lorimer 2008). Second, in the time that has passed since the original search, greatly increased computational power for acceleration searches has become feasible at a reasonable cost. Finally, a new high bandwidth (800 MHz) backend with fast sampling capability became available at the telescope (the West Virginia Ultimate Pulsar Processing Instrument, or WUPPI; see, e.g., Mickaliger et al. 2012). Sub-millisecond pulsars are in principle detectable with this instrument, and the wide bandwidth and many narrow frequency channels allow radio frequency interference (RFI) to be more easily recognized and excised. The 8-bit sampling and polyphase filterbank of WUPPI also mitigated RFI and reduced digitization loss compared to the 1-bit sampling in the Crawford et al. (2000) search. The higher central observing frequency (1200 $\mathrm{MHz}$ versus $610 \mathrm{MHz}$ for the previous survey) also helped reduce pulse broadening from interstellar dispersion and scattering. Table 2 summarizes the different observing parameters for the two surveys. For our observing system, the dispersion

\footnotetext{
5 http://www.cv.nrao.edu/nvss/NVSSlist.shtml

6 http://www.gb.nrao.edu/43m/

${ }^{7} \mathrm{http}: / /$ www.cv.nrao.edu/ sransom/presto

${ }^{8} \mathrm{http}: / /$ sigproc.sourceforge.net
} 


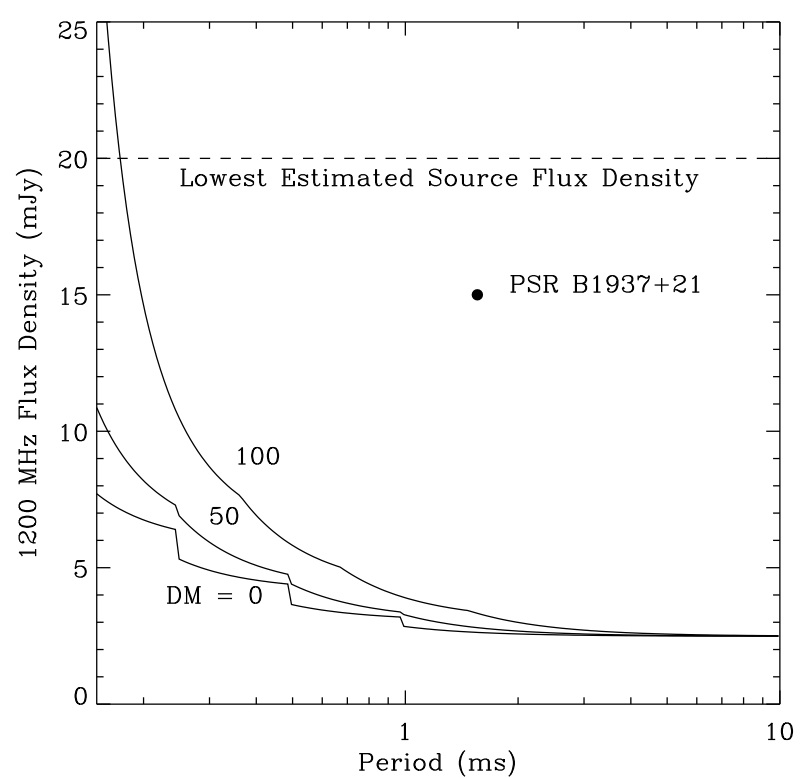

FIG. 1.- Sensitivity curves for our pulsar survey for assumed DMs of 0,50 , and $100 \mathrm{pc} \mathrm{cm}^{-3}$ and a $5 \%$ intrinsic pulsed duty cycle. Each curve represents the flux density as a function of spin period that would result in a $S / N$ of 7 in the Fourier spectrum in the search. The dashed line at $20 \mathrm{mJy}$ indicates the lowest estimated flux density of the 75 sources at $1200 \mathrm{MHz}$. The sensitivity baseline was empirically determined by scaling a $\mathrm{S} / \mathrm{N}=33$ blind detection of PSR B1937+21, which has a DM of $71 \mathrm{pc} \mathrm{cm}^{-3}$ and an estimated 1200 $\mathrm{MHz}$ flux density of $15 \mathrm{mJy}$. Not included here is the additional selection criterion that Fourier candidates must have $P \geq 0.5 \mathrm{~ms}$ to be considered, which effectively restricts our sensitivity to $P \geq 0.5 \mathrm{~ms}$.

broadening within channels was $\sim 0.1 \mathrm{~ms}$ for $\mathrm{DM}=100 \mathrm{pc}$ $\mathrm{cm}^{-3}$. This value is comparable to the previous Jodrell Bank search observations which had narrower channels but also a lower central observing frequency (Crawford et al. 2000). As seen in Figure 1, dispersion broadening does not significantly reduce the sensitivity of our search even at very low periods ( $P \lesssim 1 \mathrm{~ms}$ ). Our survey maintains good sensitivity to submillisecond pulsars for dispersion measures (DMs) between 0 and $100 \mathrm{pc} \mathrm{cm}^{-3}$, the range which is most relevant for our survey.

Apart from the effects of acceleration, the presence of orbital companions can significantly affect detectability through eclipsing by the companion. Material blown off of a companion star by the pulsar wind can obscure the pulsar signal in such cases (Tavani 1991; Hessels et al. 2006). This has been observed for a number of MSPs with non-degenerate companions, including PSR J1748-2446ad (Hessels et al. 2006) and PSR J1740-5340 (D'Amico et al. 2001), both of which are located in globular clusters and are eclipsed for $\sim 40 \%$ of their orbit. The number of "black widow" (Fruchter et al. 1988) and "redback" (Archibald et al. 2009) eclipsing pulsar systems in the Galaxy has also recently grown (see Roberts 2012 for a summary). These pulsars are in tight binary systems with eclipse fractions that can vary with frequency and which can be quite large. As mentioned above, eclipsing systems may preferentially harbor very rapidly spinning pulsars (Hessels 2008), making sub-millisecond pulsar systems even more likely to be missed. Such systems might be discovered by re-observing them at a different epoch when they are not in eclipse. Time-dependent effects such as diffractive scintillation or sporadic radio emission from the neutron star may also have previously prevented detection of some of our sources. The much larger observing bandwidth in the search described here helps mitigate diffractive scintillation effects (see Section 5 for more details).

For these reasons we re-performed a search of these targets using the 43-m Telescope and WUPPI backend. Details of our observational method are provided in Section 3, followed by a description of our data reduction techniques for both the periodicity search and the single pulse search in Section 4. The results of our search are discussed in Section 5, and our conclusions are presented in Section 6.

\section{OBSERVATIONS}

Observations of the 75 sources were conducted with the 43-m Telescope and WUPPI backend between February 2010 and August 2011. We observed each source at a center frequency of $1200 \mathrm{MHz}$ in two orthogonal linear polarizations for a total of $900 \mathrm{~s}$ per source. A bandwidth of $800 \mathrm{MHz}$ was split into 4096 frequency channels, and each channel was 8-bit sampled every $61.44 \mu \mathrm{s}$. Data were recorded directly to disk in PSRFITS format (Hotan et al. 2004). We determined an empirical flux density limit to pulsed emission for the search using a $900 \mathrm{~s}$ test observation of the bright MSP PSR B1937+21. With a DM of $71 \mathrm{pc} \mathrm{cm}^{-3}$ and a period of $1.56 \mathrm{~ms}$, PSR B1937+21 is similar to the rapidly spinning pulsars we hoped to detect. According to the ATNF catalog (Manchester et al. 2005) ${ }^{9}$, PSR B1937+21 has a flux density of $10 \mathrm{mJy}$ at $1400 \mathrm{MHz}$ and a spectral index of $\alpha=-2.6$. This corresponds to a flux density of $15 \mathrm{mJy}$ at $1200 \mathrm{MHz}$, our central observing frequency. The weakest of our 75 target sources had a flux density of $15 \mathrm{mJy}$ at $1400 \mathrm{MHz}$ (see Table 1). Assuming a typical value of $\alpha=-1.6$ for pulsars (Lorimer et al. 1995), this scales to $20 \mathrm{mJy}$ at $1200 \mathrm{MHz}$. A $900 \mathrm{~s}$ observation of PSR B1937+21 yielded a clear detection of the pulsed emission in the Fourier search with a signalto-noise ratio $(\mathrm{S} / \mathrm{N})$ of 33 in the spectrum. Given the same observing configuration and integration time, the $\mathrm{S} / \mathrm{N}$ of our weakest source would be expected to have an $\mathrm{S} / \mathrm{N}$ of 44 in the absence of time-dependent variability in intensity or Fourier bin drift due to acceleration (see Section 4.1). Assuming a $\mathrm{S} / \mathrm{N}$ threshold of 7 for detectability, it is clear that even our weakest source should be well above the limiting flux density of our observations if it is a pulsar (see Figure 1).

Each set of observations in an observing session was preceded by an approximately 90 s observation of a known bright pulsar in order to ensure that the telescope and observing software were functioning properly.

\section{DATA REDUCTION AND ANALYSIS}

We searched each data set for both fast periodic signals and single dispersed pulses at a range of DMs. The single pulse search was employed specifically to detect transient objects that would not appear in the periodicity search, and the periodicity search included a search for accelerated signals to account for binary motion. We describe these searches below.

\subsection{Periodicity Search}

We performed the periodicity search using PRESTO. Each observation was first checked for bright narrowband and undispersed short-duration signals, indicative of terrestrial RFI. Corrupted samples in the time-frequency array were replaced by median value powers. Additionally, we flagged several bands in the frequency range in which RFI was known to be persistent. On average, we masked approximately $10 \%$ of

\footnotetext{
${ }^{9}$ http://www.atnf.csiro.au/people/pulsar/psrcat
} 


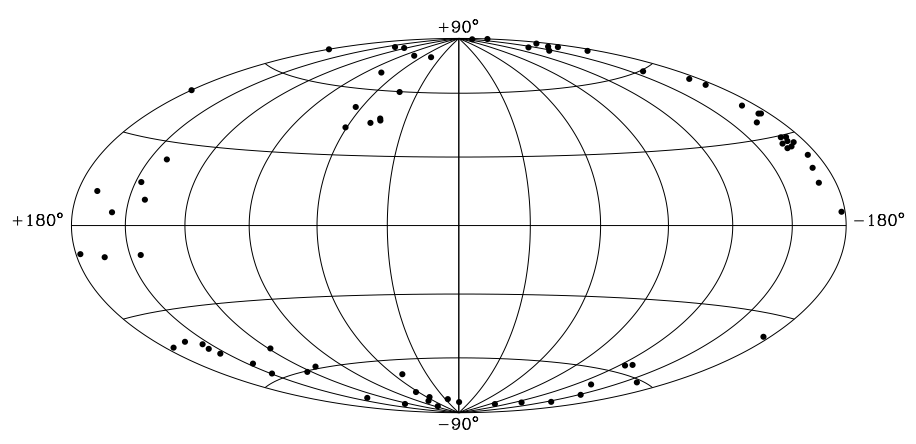

FIG. 2.- Aitoff projection plot of the sky positions of the 75 unidentified sources surveyed plotted in Galactic coordinates. The Galactic plane runs horizontally and bisects the plot. Most of the sources are located far from the Galactic plane, where there is less ISM plasma, and subsequently the maximum expected DMs are smaller. From the NE2001 model of Cordes \& Lazio (2002), the estimated maximum DM along the line of sight for all but 8 of the 75 sources is expected to be less than $100 \mathrm{pc} \mathrm{cm}^{-3}$ (Cordes \& Lazio 2002).

the total data in each beam. We dedispered the data at 401 evenly spaced trial DMs between 0 and $100 \mathrm{pc} \mathrm{cm}^{-3}$, corresponding to a step size of $0.25 \mathrm{pc} \mathrm{cm}^{-3}$. We chose this DM range since the NE2001 model of the Galactic electron distribution (Cordes \& Lazio 2002) indicates that all but 8 of the 75 sources observed are expected to have a maximum possible DM contribution along the line of sight of less than 100 pc cm${ }^{-3}$, while the remaining 8 sources are not anticipated to have maximum DMs much greater than this. This is due to their location at relatively large Galactic latitudes away from the Galactic plane (see Figure 2), where there is generally less Galactic plasma. The DM spacing was slightly larger than the ideal spacing of $\leq 0.18 \mathrm{pc} \mathrm{cm}^{-3}$ (see the single pulse search described in Section 4.2), but it still maintained sensitivity to sub-millisecond pulsars while making the Fourier search computationally feasible.

Each dedispersed time series was Fourier transformed to produce a power spectrum, and each spectrum was subsequently high-pass filtered in order to remove any slowly varying noise contribution (red noise). Each spectrum was also harmonically summed in order to enhance signals with significant integer harmonic content (e.g., Taylor \& Huguenin 1969). We performed a search on each harmonically summed spectrum, with a $\mathrm{S} / \mathrm{N}$ threshold of 7 and a searchable modulation frequency range between $1600 \mathrm{~Hz}(100 \mathrm{~Hz}$ x 16 integer harmonics, corresponding to pulsars with fundamental periods of $10 \mathrm{~ms}$ ) and $10000 \mathrm{~Hz}$. This restricted our full search sensitivity to pulsars with $P<10 \mathrm{~ms}$, which encompassed our period range of interest. However, pulsars with periods greater than this may still have been detectable if they had harmonics present above $100 \mathrm{~Hz}$.

We conducted an acceleration search to account for any binary acceleration, which causes a drift of the signal in phase over time and a decrease in $\mathrm{S} / \mathrm{N}$. Our search resulted in no sensitivity loss for the acceleration range $\pm 2.3 \mathrm{~m} \mathrm{~s}^{-2}(P / 1 \mathrm{~ms})$, where $P$ is the candidate spin period. For comparison, this search would have ensured full sensitivity to most (154 out of 167) known binary radio pulsars while they were undergoing maximum line-of-sight acceleration in their orbits (see Figure 3). This set of 167 pulsars was taken from entries in the ATNF catalog which had measured values for the projected semi-major axis and binary orbital period (see below), but it did not include any binary systems recently discovered in the High Time Resolution Universe (HTRU; Keith et al. 2010)

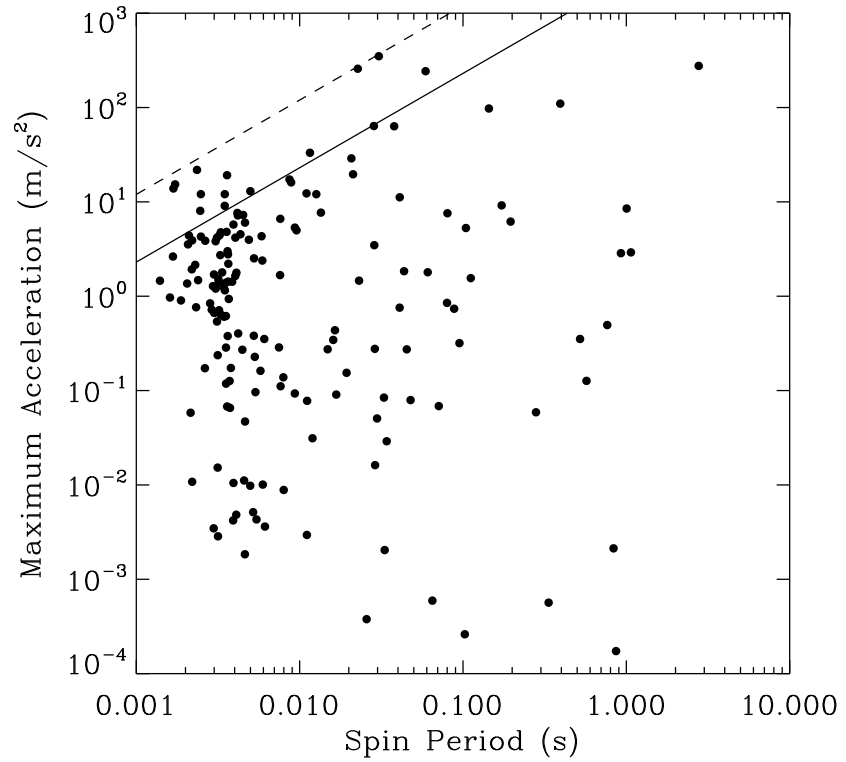

FIG. 3.- Maximum line-of-sight acceleration versus spin period for 167 known binary radio pulsars in the ATNF catalog with measured projected semi-major axis values and binary orbital periods. The maximum acceleration was calculated using the method described by Freire et al. (2001). The solid line represents the acceleration search range $\pm 2.3 \mathrm{~m} \mathrm{~s}^{-2}(P / 1 \mathrm{~ms})$, for which full sensitivity was maintained in our search. All but 13 of the pulsars fall below this cutoff, indicating that our search range would be sufficient to detect most known pulsars in binary systems even if they were observed while undergoing maximum acceleration in their orbits. The dashed line indicates the acceleration search range $\pm 12 \mathrm{~m} \mathrm{~s}^{-2}(P / 1 \mathrm{~ms})$. This acceleration search range would provide full sensitivity to all 167 pulsars shown in the plot. Our search was not performed out to this range owing to computational limitations. However, given the large flux densities of our sources compared to our sensitivity limit, even such extremely accelerated pulsars would still be expected to be detectable with a S/N above 7 in our search in the absence of flux variability (see Section 4.1).

or Pulsar Arecibo L-Band Feed Array (PALFA; Cordes et al. 2006) surveys.

To calculate the maximum line-of-sight accelerations for these 167 pulsars, we used an expression derived by Freire et al. (2001) in which the acceleration of a pulsar in a binary system is described as a function of observable parameters. From this equation, the maximum possible acceleration $A_{\max }$ is

$$
A_{\text {max }}=\frac{4 \pi^{2} a(1+e)^{2}}{P_{b}^{2}\left(1-e^{2}\right)^{2}},
$$

where $a$ is the projected semi-major axis of the pulsar's orbit, $P_{b}$ is the orbital period, and $e$ is the orbital eccentricity.

For the sample of 167 binary radio pulsars, if no eccentricity $e$ was measured in the ATNF catalog, we assumed $e=0$ (a perfectly circular orbit) for the calculation. As seen in Figure 3, an acceleration search range of $\pm 12 \mathrm{~m} \mathrm{~s}^{-2}(P / 1 \mathrm{~ms})$ would ensure full sensitivity to all known binary radio pulsars in the sample undergoing maximum acceleration. However, the computational time required to perform a search of our 75 sources with this full acceleration range was excessive. Furthermore, the relatively large flux densities of our sources ensured that pulsars with accelerations near the highest values in this sample of 167 would probably still have been detectable even if the $\mathrm{S} / \mathrm{N}$ were reduced by acceleration bin drift. The amount of Fourier bin drift of a pulse depends linearly on the acceleration. For a acceleration of $\pm 12 \mathrm{~m} \mathrm{~s}^{-2}(P / 1 \mathrm{~ms})$, the 
$\mathrm{S} / \mathrm{N}$ would be reduced by a factor of 5.2 when searching only out to an acceleration of $\pm 2.3 \mathrm{~m} \mathrm{~s}^{-2}(P / 1 \mathrm{~ms})$. Since we expect our weakest source to have a $\mathrm{S} / \mathrm{N}$ of 44 in the absence of time-dependent variability in intensity (see Section 3), the smallest $\mathrm{S} / \mathrm{N}$ we would expect from an extremely accelerated pulsar is $\geq 8$, which is still above our survey detection limit of 7 (see Figure 1).

The acceleration search produced a list of candidate signals for each trial DM with periods and spectral $\mathrm{S} / \mathrm{N}$ values. These candidate files from different DM trials for a source were combined into a master list. We subsequently removed candidates if they were deemed unlikely to be pulsars (see below) and also removed duplicates and harmonically related signals. The heuristics used in keeping good candidates included the requirements that the candidate have its maximum signal at a DM greater than $1 \mathrm{pc} \mathrm{cm}^{-3}$ and that it have a period $P \geq 0.5 \mathrm{~ms}$. The result was a list of the best pulsar candidates for a source ranked by $\mathrm{S} / \mathrm{N}$. We then dedispersed and folded the raw data at periods and DMs near the candidate values. We inspected the resulting plots by eye to see if promising features were present, indicating that a confirmation observation was warranted.

We tested this method with a 900 s observation of PSR B1937+21 using the same observing system as was used for the 75 survey sources. We successfully detected the pulsar in the search with a DM and period consistent with its catalog values.

\subsection{Single Pulse Search}

We performed a search for single pulses in the data using SIGPROC. Data from each source were dedispersed using 713 trial DMs with variable spacing, with the DMs ranging between 0 and $100 \mathrm{pc} \mathrm{cm}^{-3}$. The maximum spacing between $\mathrm{DM}$ trials in this range was $0.18 \mathrm{pc} \mathrm{cm}^{-3}$. We chose the spacing to ensure that the dispersion smearing due to an offset in DM did not greatly exceed the dispersion smearing within the frequency channels at that DM. We checked each dedispersed time series for pulses having a range of widths using a boxcar smoothing technique described by Cordes \& McLaughlin (2003). We tested this technique on a $900 \mathrm{~s}$ observation of the Crab pulsar (PSR B0531+21), and we detected single pulses from the Crab easily. The method was also tested on the same 900 s observation of PSR B1937+21 that was used to test the periodicity search technique. No single pulses were detected in that search, which had a minimum $\mathrm{S} / \mathrm{N}$ threshold of 5. While PSR B1937+21 and the Crab pulsar have similar giant pulse emission rates (Cognard et al. 1996) as well as comparable $1400 \mathrm{MHz}$ flux densities, the giant pulses emitted by PSR B1937+21 are much narrower than the survey's sampling time of $61.44 \mu \mathrm{s}$ (the pulse widths are on the order of a few microseconds; see Figure 1 of Cognard et al. 1996). These narrow pulses are smeared out within each time sample, leading to a significant reduction in the $\mathrm{S} / \mathrm{N}$. Thus, it is unlikely that such narrow pulses would be detected in our single pulse search. This also indicates that our search is not sensitive to extremely narrow single pulses or bursts (a few $\mu \mathrm{s}$ or less in width) from our target sources.

\subsection{Follow-up Observations}

One candidate from the periodicity search showed merit and warranted re-observation at the telescope. We conducted a confirmation observation using the same observing system and integration time (900 s) as the original observation. The data were dedispersed and folded at a range of DMs and periods near the candidate period and DM to see if the same signal was re-detected. We also performed a blind periodicity search and single pulse search on the confirmation data. The signal was not confirmed.

\section{RESULTS AND DISCUSSION}

We confirmed no dispersed pulsations or transient bursts from any of the target sources in either the periodicity search or the single pulse search. Below we consider possible factors that could have led to the non-detections if these sources were pulsars.

It is unlikely that diffractive scintillation caused us to miss detections in most cases. This can be shown using several different assumptions to calculate the expected scintillation bandwidths for these sources. First, an expression given by Cordes et al. (1985) gives an estimated typical scintillation bandwidth $\Delta \nu_{\text {scint }}$ in $\mathrm{MHz}$ (see also Equation 2 in Crawford et al. 2000):

$$
\Delta \nu_{\text {scint }} \simeq 11 \nu_{c}^{4.4} d^{-2.2}
$$

Here $\nu_{c}$ is the central observing frequency in $\mathrm{GHz}$ and $d$ is the source distance in kpc. For $d=0.2 \mathrm{kpc}$ and $\nu_{c}=1.2$ $\mathrm{GHz}, \Delta \nu_{\text {scint }}$ is comparable to our observing bandwidth of $800 \mathrm{MHz}$. Thus, sources with $d<0.2 \mathrm{kpc}$ would be expected to occasionally be undetectable in our search owing to diffractive scintillation. Note that this does not take into account the large degree of variability in $\Delta \nu_{\text {scint }}$ that exists as a function of sky position. A calculation of scintillation bandwidths for the 75 sky positions of our sources using the NE2001 model Cordes \& Lazio (2002) gives similar results: for a frequency of $1200 \mathrm{MHz}$ and an assumed distance $d=0.2 \mathrm{kpc}$, we find that only 3 of the 75 sources have $\Delta \nu_{\text {scint }}<800 \mathrm{MHz}$ (where we have assumed a Kolmogorov scaling dependence of $\nu^{4.4}$ for $\left.\Delta \nu_{\text {scint }}\right)$. This number increases to 58 and 75 for $d=0.5$ and $1.0 \mathrm{kpc}$, respectively. Thus, it appears that the range 0.2 to $0.5 \mathrm{kpc}$ represents a critical distance below which scintillation bandwidths rapidly increase for these sources and where they can exceed the $800 \mathrm{MHz}$ observing bandwidth. We can also estimate a typical source distance using several assumptions. The median $1400 \mathrm{MHz}$ flux density of our 75 target sources is $S=51 \mathrm{mJy}$, and the median value of the $1400 \mathrm{MHz}$ pseudoluminosity for known recycled radio pulsars in the ATNF pulsar catalog (Manchester et al. 2005) is $L \sim 2 \mathrm{mJy} \mathrm{kpc}^{2}$. The pseudo-luminosity $L$ is defined according to $L=S d^{2}$ (Lorimer $\&$ Kramer 2004). Using these values for $L$ and $S$, we obtain a characteristic source distance of $d \sim 0.2 \mathrm{kpc}$, which is close to the critical distance described above. The observed median $L$ value used here may be overestimated if the luminosity distribution of the underlying population of recycled pulsars extends well below the observed lower limit. In this case, the derived characteristic source distance $d$ would be reduced as well. In any case, even if $\Delta \nu_{\text {scint }}$ were to exceed $800 \mathrm{MHz}$ for every source, this would still not prevent the detection of the majority of our sources. The likelihood of detection of a source in this case is an exponential function dependent on the source flux density and flux density limit of the search observation. The total number of sources $n$ expected to be detectable is the sum of these individual detection likelihoods over all sources (see Crawford et al. (2000) for a description and similar calculation), 


$$
n=\sum_{i=1}^{75} e^{-S_{i} / S_{\min }}
$$

where $S_{\min }$ is the $1200 \mathrm{MHz}$ flux density limit of the survey ( $\sim 3 \mathrm{mJy}$; see Figure 1$)$ and the sum is taken over all 75 sources having $1200 \mathrm{MHz}$ flux densities $S_{i}$ (which we estimated using the cataloged $1400 \mathrm{MHz}$ values and a typical pulsar spectral index of $\alpha=-1.6$ in those cases where $\alpha$ could not be measured). This calculation shows that $n=71$ (out of 75 ) sources should still be detectable in our survey. Therefore, diffractive scintillation is not expected to be a dominant reason for the non-detection of these sources if they are pulsars.

It is possible that some of these sources could be binary pulsars that are being eclipsed and that the pulsar signal was masked at the time of observation by material ablated from the companion by the pulsar wind (e.g., black widows or redbacks). As mentioned previously, very rapidly spinning pulsars may exist more commonly in eclipsing systems (Hessels 2008). Since the obscuration is frequency-dependent, with detectability decreasing at lower observing frequencies, this effect could be mitigated by conducting higher-frequency observations in the future. It is not likely that binary acceleration is a significant factor in any of our non-detections. Our range of trial accelerations in the periodicity search was sufficient to maintain sensitivity to even the most highly accelerated radio pulsar systems currently known (see Figure 3).

Only 8 of our 75 sources had maximum estimated DMs from the NE2001 model that were greater than $100 \mathrm{pc} \mathrm{cm}^{-3}$ (Cordes \& Lazio 2002). Since we searched DMs up to 100 $\mathrm{pc} \mathrm{cm}{ }^{-3}$, it is possible that dispersive smearing rendered pulsations from some of these sources undetectable. However, since the sources in our sample are very bright relative to our survey detection limit, they are also likely to be relatively close (see the estimated distance discussion above), with corresponding DMs that are much smaller than the maximum DM. Thus, dispersive smearing is unlikely to be a significant factor. Using the known recycled pulsar population as a guide, the estimated characteristic distance to the sources is small ( $d$ $\sim 0.2 \mathrm{kpc}$; see above). For this distance, the NE2001 model indicates that the corresponding DMs are between about 1 and $5 \mathrm{pc} \mathrm{cm}^{-3}$ for all 75 sources. Our candidate selection criteria in the search included keeping Fourier candidates having DM $>1 \mathrm{pc} \mathrm{cm}^{-3}$. Nevertheless, pulsar signals at such low DMs are at risk of being flagged as RFI in the processing. For several of our sources for which there was a significant amount of RFI present in the data, removal of a low-DM pulsar signal during the RFI excision process is a possibility. RFI in these beams also affected the single pulse search, and any low-DM transient signals from the target sources may have been removed along with RFI.

If some of these sources are transient objects, such as RRATs (McLaughlin et al. 2006) or intermittent or nulling pulsars (e.g., Kramer et al. 2006; Wang et al. 2007), it is possible that they were simply not active at the time of observation. The discovery of the intermittent emission behavior of the supposedly ordinary pulsar PSR B1931+24 by Kramer et al. (2006) suggests that many more such objects could have been missed in previous pulsar searches. In this case, the chances of detecting pulsations from our target sources would increase with additional observations, preferably with longer observing times.

\section{CONCLUSIONS}

No new pulsars or transient objects were discovered in the survey, and the question of what the unidentified target sources are remains unanswered. Below we outline some speculative possibilities.

Some of these sources may be active galactic nuclei (AGN) that will be identified in the future. While only $\sim 10 \%$ of known quasars and BL Lac objects are more than 5\% linearly polarized (Han \& Tian 1999), this does not completely discount extragalactic sources as a possibility. In fact, as seen in Table 1, 16 of the 92 previously unidentified sources searched by Crawford et al. (2000) have been been identified as extragalactic objects and are no longer candidate pulsars. The generally shallower spectral indices seen for our target sources in Table 1 relative to the steeper average observed for the radio pulsar population also suggests this as a possibility.

Another possibility is that some of these sources might be radio-emitting white dwarfs (WDs). Persistent radio emission has been observed from several WD systems. Chanmugam \& Dulk (1982) were the first to observe radio emission from AM Her, a magnetic cataclysmic variable (MCV). AM Her is in a short-period binary orbit and has a strong magnetic field. Radio emission was subsequently observed from two other MCVs: AE Aqr (Bookbinder \& Lamb 1987; Bastian et al. 1988) and AR UMa (Mason \& Gray 2007). However, radio searches of a range of CV subclasses indicates that they are not generally radio detected (Cordova et al. 1983; Chanmugam 1987; Mason \& Gray 2007). It is worth noting that Mason \& Gray (2007) observed the FIRST source $\mathrm{J} 1023+0038$ to search for radio emission. At the time, this source was tentatively identified as a CV associated with a FIRST radio source (Bond et al. 2002). Mason \& Gray (2007) found no radio emission from $\mathrm{J} 1023+0038$, but the source was subsequently detected and identified as the first redback radio pulsar system discovered in the Galactic field (Archibald et al. 2009). This highlights the importance of repeated observations of undetected radio sources.

The observed radio sources might also be radio pulsars having closely aligned spin and magnetic axes. A study by Tauris \& Manchester (1998) found that there is a tendency for the magnetic and spin axes to become aligned as pulsars age. Using pulsar polarization data sets from Rankin (1993) and Gould (1994), they found that this alignment occurs on a timescale of $\sim 10^{7}$ yr. More recent work by Young et al. (2010) using Candy-Blair evolution models (Candy \& Blair 1983, 1986; Jones 1976) also shows progressive alignment occurring, but on a time-scale of $\sim 10^{6} \mathrm{yr}$. Such aligned rotators would be hard or impossible to detect in traditional pulsar searches owing to their decreased (or non-existent) modulation (Tauris \& Manchester 1998). This is illustrated in Figure 1 of Young et al. (2010), which shows that in cases where the emission cone encompasses the spin axis, one would not expect to see a modulated signal. If our target sources are indeed unmodulated radio pulsars with aligned magnetic and spin axes, then these models indicate that they are likely to be old pulsars. In this case, the shallower spectral indices of these older pulsars (see Table 1) relative to the general radio pulsar population would need to be explained.

If these unidentified sources are rapidly rotating pulsars with modulated radio emission, the effects of diffractive scintillation, dispersive smearing, and binary acceleration are unlikely to have prevented the detection of a large majority of the targets in our search. However, we cannot rule out eclipsing, intermittent emission, nulling, or RFI as possibly important factors. Just as Fermi sources must be searched multiple 
times before declaring that no radio pulsar is present (Ray et al. 2012), repeated observations of our targets may be required to reach the same conclusion.

The National Radio Astronomy Observatory (NRAO) is a facility of the National Science Foundation operated under cooperative agreement by Associated Universities, Inc. Funding for some of the equipment used was provided by West Virginia EPSCoR and Research Corporation. D.R.S. was sup- ported at the NRAO by the Research Experience for Undergraduates program, which is funded by the National Science Foundation. This research has made use of the SIMBAD database, operated at CDS, Strasbourg, France. We thank Maura McLaughlin for valuable suggestions which helped to optimize the periodicity search algorithm, and we thank an anonymous referee for recommendations that improved the paper. D.R.S. also wishes to thank the staff of the Green Bank Observatory for their hospitality during her time there.

\section{REFERENCES}

Archibald, A. M., Stairs, I. H., Ransom, S. M., et al. 2009, Science, 324, 1411 Bastian, T. S., Dulk, G. A., \& Chanmugam, G. 1988, ApJ, 324, 431

Becker, R. H., White, R. L., \& Helfand, D. J. 1995, ApJ, 450, 559

Bildsten, L. 1998, ApJ, 501, L89

Blake, C., \& Wall, J. 2002, Nature, 416, 150

Bond, H. E., White, R. L., Becker, R. H., \& O’Brien, M. S. 2002, PASP, 114 1359

Bookbinder, J. A., \& Lamb, D. Q. 1987, ApJ, 323, L131

Candy, B. N., \& Blair, D. G. 1983, MNRAS, 205, 281

Candy, B. N., \& Blair, D. G. 1986, ApJ, 307, 535

Chanmugam, G., \& Dulk, G. A. 1982, ApJ, 255, L107

Chanmugam, G. 1987, Ap\&SS, 130, 53

Cognard, I., Shrauner, J. A., Taylor, J. H., \& Thorsett, S. E. 1996, ApJ, 457, L81

Condon, J. J., Cotton, W. D., Greisen, E. W., et al. 1998, AJ, 115, 1693

Cook, G. B., Shapiro, S. L., \& Teukolsky, S. A. 1994, ApJ, 423, L117

Cordes, J. M., \& Lazio, T. J. W. 2002, arXiv:astro-ph/0207156

Cordes, J. M., \& McLaughlin, M. A. 2003, ApJ, 596, 1142

Cordes, J. M., Weisberg, J. M., \& Boriakoff, V. 1985, ApJ, 288, 221

Cordes, J. M., Freire, P. C. C., Lorimer, D. R., et al. 2006, ApJ, 637, 446

Cordova, F. A., Hjellming, R. M., \& Mason, K. O. 1983, PASP, 95, 69

Crawford, F., Kaspi, V. M., \& Bell, J. F. 2000, AJ, 119, 2376

Crawford, F. 2009, ApJ, 692, 887

D'Amico, N., Possenti, A., Manchester, R. N., et al. 2001, ApJ, 561, L89

Douglas, J. N., Bash, F. N., Bozyan, F. A., Torrence, G. W., \& Wolfe, C. 1996, AJ, 111, 1945

Freire, P. C., Kramer, M., \& Lyne, A. G. 2001, MNRAS, 322, 885

Fruchter, A. S., Stinebring, D. R., \& Taylor, J. H. 1988, Nature, 333, 237

Gould, D. M. 1994, Ph.D. Thesis, Jodrell Bank Obs.

Gregory, P. C., \& Condon, J. J. 1991, ApJS, 75, 1011

Griffith, M. R., \& Wright, A. E. 1993, AJ, 105, 1666

Haensel, P., Lasota, J. P., \& Zdunik, J. L. 1999, A\&A, 344, 151

Hales, S. E. G., Riley, J. M., Waldram, E. M., Warner, P. J., \& Baldwin, J. E. 2007, MNRAS, 382, 1639

Han, J. L., \& Tian, W. W. 1999, A\&AS, 136, 571

Hessels, J. W. T., Ransom, S. M., Stairs, I. H., et al. 2006, Science, 311, 1901

Hessels, J. W. T. 2008, American Institute of Physics Conference Series, 1068,130

Ho, W. C. G., Maccarone, T. J., \& Andersson, N. 2011, ApJ, 730, L36

Hotan, A. W., van Straten, W., \& Manchester, R. N. 2004, Publications of the Astronomical Society of Australia, 21, 302
Jones, P. B. 1976, Ap\&SS, 45, 369

Keith, M. J., Jameson, A., van Straten, W., et al. 2010, MNRAS, 409, 619

Kramer, M., Lyne, A. G., O'Brien, J. T., Jordan, C. A., \& Lorimer, D. R. 2006, Science, 312, 549

Lattimer, J. M., \& Prakash, M. 2004, Science, 304, 536

Lorimer, D. R., Yates, J. A., Lyne, A. G., \& Gould, D. M. 1995, MNRAS, 273, 411

Lorimer, D. R., \& Kramer, M. 2004, Handbook of Pulsar Astronomy (Cambridge: Cambridge Univ. Press)

Lorimer, D. R. 2008, Living Reviews in Relativity, 11, 8

Lyne, A. G., \& Manchester, R. N. 1988, MNRAS, 234, 477

Manchester, R. N., Hobbs, G. B., Teoh, A., \& Hobbs, M. 2005, AJ, 129, 1993

Mason, P. A., \& Gray, C. L. 2007, ApJ, 660, 662

McLaughlin, M. A., Lyne, A. G., Lorimer, D. R., et al. 2006, Nature, 439, 817

Mickaliger, M. B., McLaughlin, M. A., Lorimer, D. R., et al. 2012, ApJ, 760, 64

Nolan, P. L., Abdo, A. A., Ackermann, M., et al. 2012, ApJS, 199, 31

Patruno, A., Haskell, B., \& D’Angelo, C. 2012, ApJ, 746, 9

Possenti, A., Colpi, M., D’Amico, N., \& Burderi, L. 1998, ApJ, 497, L97

Rankin, J. M. 1993, ApJ, 405, 285

Ransom, S. M. 2001, Ph.D. Thesis, Harvard University

Ransom, S. M., Eikenberry, S. S., \& Middleditch, J. 2002, AJ, 124, 1788

Ray, P. S., Abdo, A. A., Parent, D., et al. 2012, 2011 Fermi Symposium, arXiv: 1205.3089

Ray, P. S., Ransom, S. M., Cheung, C. C., et al. 2013, ApJ, 763, L13

Roberts, M. S. E. 2012, Proceedings of IAUS 291 "Neutron Stars and Pulsars: Challenges and Opportunities after 80 years," J. van Leeuwen (ed.), arXiv: 1210.6903

Staelin, D. H., \& Reifenstein, E. C., III 1968, Science, 162, 148

Tauris, T. M., \& Manchester, R. N. 1998, MNRAS, 298, 625

Tauris, T. M. 2012, Science, 335, 561

Tavani, M. 1991, Nature, 351, 39

Taylor, J. H., \& Huguenin, G. R. 1969, Nature, 221, 816

Wagoner, R. V. 1984, ApJ, 278, 345

Wang, N., Manchester, R. N., \& Johnston, S. 2007, MNRAS, 377, 1383

Young, M. D. T., Chan, L. S., Burman, R. R., \& Blair, D. G. 2010, MNRAS, 402, 1317

TABLE 1

NVSS RADIO SOURCE TARGETS

\begin{tabular}{cccccccc}
\hline \hline NVSS Source & $\begin{array}{c}\alpha(\mathrm{J} 2000.0) \\
(\mathrm{h} \mathrm{m} \mathrm{s})\end{array}$ & $\begin{array}{c}\delta(\mathrm{J} 2000.0) \\
(\mathrm{d} \mathrm{m} \mathrm{s})\end{array}$ & $\begin{array}{c}S_{1400}{ }^{\mathrm{a}} \\
(\mathrm{mJy})\end{array}$ & $\begin{array}{c}\text { Lin. Poln }^{\mathrm{b}} \\
(\%)\end{array}$ & Source ID $^{\mathrm{c}}$ & $\alpha^{\mathrm{d}}$ & Survey $^{\mathrm{e}}$ \\
\hline $\mathrm{J} 000240-195252$ & 000240.96 & -195252.3 & 60 & 9 & & & \\
$\mathrm{~J} 000404-114858$ & 000404.90 & -114858.4 & 459 & 6 & $\mathrm{~B}$ & & \\
$\mathrm{~J} 001109-225458$ & 001109.91 & -225458.5 & 38 & 10 & & & \\
$\mathrm{~J} 001444-280047$ & 001444.06 & -280047.3 & 54 & 14 & & $-0.32(11)$ & $\mathrm{PMN}$ \\
$\mathrm{J} 002330-215537$ & 002330.21 & -215537.6 & 137 & 8 & & & \\
$\mathrm{~J} 002449+030834$ & 002449.37 & +030834.7 & 69 & 9 & & \\
$\mathrm{~J} 002651-111252$ & 002651.45 & -111252.4 & 169 & 8 & $\mathrm{Q}$ & \\
$\mathrm{J} 002702-303032$ & 002702.07 & -303032.0 & 24 & 14 & & \\
$\mathrm{~J} 003233-264917$ & 003233.03 & -264917.6 & 135 & 7 & $\mathrm{Q}$ & \\
$\mathrm{J} 003708-232340$ & 003708.81 & -232340.5 & 67 & 6 & & \\
$\mathrm{~J} 004021+132937$ & 004021.80 & +132937.9 & 34 & 10 & & \\
$\mathrm{~J} 005151+022944$ & 005151.30 & +022944.2 & 15 & 21 & & \\
$\mathrm{~J} 005410-175413$ & 005410.78 & -175413.0 & 30 & 11 & & \\
$\mathrm{~J} 005736+134145$ & 005736.44 & +134145.4 & 65 & 9 & $\mathrm{G}$ & & \\
$\mathrm{J} 010711-121123$ & 010711.79 & -121123.6 & 60 & 6 & & $-0.37(13)$ & PMN \\
$\mathrm{J} 011448-321951$ & 011448.89 & -321951.7 & 124 & 16 & & \\
\hline
\end{tabular}


Schmidt et al.

TABLE 1 - Continued

\begin{tabular}{|c|c|c|c|c|c|c|c|}
\hline NVSS Source & $\begin{array}{c}\alpha(\mathrm{J} 2000.0) \\
(\mathrm{h} \mathrm{m} \mathrm{s})\end{array}$ & $\begin{array}{c}\delta(\mathrm{J} 2000.0) \\
(\mathrm{d} \mathrm{m} \mathrm{s})\end{array}$ & $\begin{array}{l}S_{1400^{\mathrm{a}}} \\
(\mathrm{mJy})\end{array}$ & $\begin{array}{l}\text { Lin. Poln } \\
(\%)\end{array}$ & Source ID ${ }^{c}$ & $\alpha^{\mathrm{d}}$ & Survey $^{\mathrm{e}}$ \\
\hline J013840-295445 & 013840.50 & -295445.9 & 46 & 10 & & & \\
\hline J014614+022208 & 014614.62 & +022208.2 & 138 & 8 & & $-0.13(9)$ & PMN \\
\hline $\mathrm{J} 014727+071502$ & 014727.77 & +071502.9 & 241 & 6 & & $-0.72(6)$ & TXS \\
\hline J015456-242233 & 015456.90 & -242233.5 & 45 & 11 & & & \\
\hline J021459+102748 & 021459.23 & +102748.8 & 29 & 13 & & & \\
\hline J021750-235456 & 021750.76 & -235456.3 & 90 & 11 & & & \\
\hline J022333+073219 & 022333.94 & +07 3219.6 & 125 & 13 & G & & \\
\hline J022340+115910 & 022340.83 & +115910.2 & 34 & 10 & & & \\
\hline J022441+135733 & 022441.85 & +135733.1 & 95 & 10 & & $-0.17(13)$ & $87 \mathrm{~GB}$ \\
\hline J023855-303202 & 023855.19 & -303202.6 & 165 & 5 & & & \\
\hline J024944+123706 & 024944.50 & +123706.3 & 261 & 6 & & $-0.65(12)$ & $87 \mathrm{~GB}$ \\
\hline J025106-174239 & 025106.22 & -174239.5 & 69 & 13 & & $-0.09(15)$ & PMN \\
\hline J025805-314627 & 025805.95 & -314627.8 & 247 & 8 & $\mathrm{Q}$ & & \\
\hline J025927+074739 & 025927.06 & +074739.2 & 834 & 5 & Q & & \\
\hline J025904+470840 & 025904.20 & +470840.4 & 108 & 9 & & $-0.58(7)$ & TXS \\
\hline J031726+060614 & 031726.85 & +06 0614.7 & 200 & 8 & & $-0.89(14)$ & PMN \\
\hline J032213-345833 & 032213.10 & -345833.2 & 49 & 7 & & & \\
\hline J032615-324324 & 032615.12 & -324324.3 & 95 & 5 & & & \\
\hline J034914+035445 & 034914.31 & +035445.4 & 150 & 8 & & $+0.30(16)$ & PMN \\
\hline J040342+644556 & 040342.80 & +64 4556.1 & 74 & 5 & & $-0.22(10)$ & $87 \mathrm{~GB}$ \\
\hline J042119+351115 & 042119.72 & +3511 15.6 & 68 & 10 & & & \\
\hline J045828+495355 & 045828.75 & +495355.8 & 19 & 13 & & & \\
\hline J050554+260625 & 050554.20 & +260625.1 & 23 & 11 & & & \\
\hline J051843+643958 & 051843.68 & +643958.0 & 28 & 13 & & & \\
\hline J060650+440140 & 060650.20 & +440140.9 & 147 & 8 & & $-0.24(5)$ & $7 \mathrm{C}$ \\
\hline J060718+291527 & 060718.95 & +291527.7 & 25 & 16 & & & \\
\hline J062052+733441 & 062052.10 & +733441.2 & 85 & 10 & & $-0.69(11)$ & $87 \mathrm{~GB}$ \\
\hline $\mathrm{J} 070120+263157$ & 070120.74 & +263157.1 & 32 & 12 & & & \\
\hline J071923+293551 & 071923.05 & +293551.8 & 18 & 13 & & & \\
\hline J073313+333151 & 073313.31 & +33 3151.8 & 19 & 14 & & & \\
\hline $\mathrm{J} 075501+301347$ & 075501.81 & +301347.4 & 51 & 12 & & $-1.13(9)$ & TXS \\
\hline J075536+334159 & 075536.71 & +334159.2 & 82 & 7 & & & \\
\hline J075752+272111 & 075752.82 & +272111.3 & 45 & 7 & & & \\
\hline J075808+392928 & 075808.84 & +392928.7 & 545 & 8 & G & & \\
\hline $\mathrm{J} 080212+312240$ & 080212.78 & +312240.7 & 86 & 10 & & & \\
\hline J080519+273736 & 080519.02 & +273736.1 & 42 & 10 & & & \\
\hline $\mathrm{J} 080601+331010$ & 080601.70 & +331010.3 & 44 & 6 & & & \\
\hline $\mathrm{J} 081040+303433$ & 081040.30 & +303433.7 & 154 & 6 & & & \\
\hline J084030+292336 & 084030.72 & +292336.8 & 18 & 14 & & & \\
\hline J084308+373816 & 084308.76 & +373816.2 & 111 & 12 & & & \\
\hline J084456+362927 & 084456.27 & +362927.5 & 50 & 7 & & & \\
\hline $\mathrm{J} 084647+374615^{\mathrm{f}}$ & 084647.43 & +374615.1 & 22 & 16 & & & \\
\hline J090305+352316 & 090305.50 & +352316.2 & 58 & 6 & & & \\
\hline J091147+334917 & 091147.77 & +334917.1 & 380 & 7 & B & & \\
\hline J092329+301106 & 092329.97 & +301106.7 & 36 & 6 & $\mathrm{Q}$ & & \\
\hline J092822+414221 & 092822.18 & +414221.9 & 98 & 12 & & $-0.95(15)$ & TXS \\
\hline J094459+380317 & 094459.21 & +380317.6 & 43 & 12 & & & \\
\hline $\mathrm{J} 100022+371844$ & 100022.29 & +37 1844.4 & 35 & 10 & & & \\
\hline $\mathrm{J} 100357+324403$ & 100357.63 & +324403.7 & 429 & 8 & Q & & \\
\hline $\mathrm{J} 101349+344550$ & 101349.59 & +344550.7 & 356 & 6 & $\mathrm{Q}$ & & \\
\hline $\mathrm{J} 103319+285121$ & 103319.66 & +285121.0 & 29 & 10 & & $0.00(17)$ & $87 \mathrm{~GB}$ \\
\hline J112612+341818 & 112612.32 & +341818.2 & 40 & 7 & & & \\
\hline J112951+362217 & 112951.56 & +362217.1 & 122 & 7 & & & \\
\hline J114523+314515 & 114523.20 & +314515.3 & 83 & 10 & & & \\
\hline $\mathrm{J} 114608+260105$ & 114608.52 & +260105.3 & 116 & 7 & & $-0.62(14)$ & $87 \mathrm{~GB}$ \\
\hline $\mathrm{J} 115043+302018$ & 115043.89 & +302018.3 & 32 & 14 & & & \\
\hline $\mathrm{J} 120125+255006$ & 120125.63 & +255006.9 & 25 & 26 & & & \\
\hline $\mathrm{J} 120144+312904$ & 120144.50 & +312904.1 & 91 & 6 & & & \\
\hline $\mathrm{J} 122004+311149$ & 122004.37 & +31 1149.0 & 29 & 10 & Q & & \\
\hline J123454+291744 & 123454.36 & +291744.2 & 446 & 9 & & $-0.52(9)$ & $7 \mathrm{C}$ \\
\hline $\mathrm{J} 123650+370603$ & 123650.94 & +370603.8 & 63 & 10 & & & \\
\hline $\mathrm{J} 124219+272156$ & 124219.75 & +272156.2 & 70 & 10 & & $-0.65(8)$ & $7 \mathrm{C}$ \\
\hline $\mathrm{J} 125124+364354$ & 125124.28 & +364354.9 & 30 & 8 & & & \\
\hline J133426+343425 & 133426.94 & +343425.8 & 49 & 8 & & & \\
\hline J134324+290358 & 134324.38 & +290358.6 & 24 & 18 & & & \\
\hline $\mathrm{J} 141440+402229$ & 141440.46 & +402229.7 & 44 & 6 & & & \\
\hline $\mathrm{J} 142658+403539$ & 142658.42 & +403539.6 & 29 & 9 & Q & & \\
\hline $\mathrm{J} 143447+380514$ & 143447.05 & +38 0514.4 & 153 & 9 & A & & \\
\hline $\mathrm{J} 145844+372022$ & 145844.77 & +372022.0 & 215 & 6 & B & & \\
\hline $\mathrm{J} 150808+281811$ & 150808.32 & +281811.2 & 77 & 7 & & & \\
\hline $\mathrm{J} 154740+395438$ & 154740.19 & +395438.4 & 131 & 15 & & & \\
\hline J160616+270930 & 160616.20 & +270930.6 & 29 & 8 & & & \\
\hline $\mathrm{J} 160950+262839$ & 160950.12 & +262839.4 & 20 & 17 & & & \\
\hline $\mathrm{J} 161827+293118$ & 161827.05 & +293118.3 & 30 & 9 & & & \\
\hline $\mathrm{J} 163552+375159$ & 163552.59 & +375159.5 & 48 & 7 & & & \\
\hline
\end{tabular}


TABLE 1 - Continued

\begin{tabular}{cccccccc}
\hline \hline NVSS Source & $\begin{array}{c}\alpha(\mathrm{J} 2000.0) \\
(\mathrm{h} \mathrm{m} \mathrm{s})\end{array}$ & $\begin{array}{c}\delta(\mathrm{J} 2000.0) \\
(\mathrm{d} \mathrm{m} \mathrm{s})\end{array}$ & $\begin{array}{c}S_{1400^{\mathrm{a}}} \\
(\mathrm{mJy})\end{array}$ & $\begin{array}{c}\text { Lin. Poln }^{\mathrm{b}} \\
(\%)\end{array}$ & Source ID $^{\mathrm{c}}$ & $\alpha^{\mathrm{d}}$ & Survey $^{\mathrm{e}}$ \\
\hline $\mathrm{J} 232102-175822$ & 232102.41 & -175822.0 & 18 & 15 & & \\
\hline
\end{tabular}

NotE. - The original set of 92 NVSS target sources from Table 1 of Crawford et al. (2000) is presented here, including 16 sources that have been more recently identified as extragalactic objects. The flux densities and polarization fractions listed were taken from the NVSS catalog browser (revision 2.18, 2004-02-03; http://www.cv.nrao.edu/nvss/NVSSlist.shtml).

${ }^{a}$ Nominal $1400 \mathrm{MHz}$ flux density from the NVSS catalog.

${ }^{\mathrm{b}}$ Linearly polarized intensity as a percentage of total source intensity, derived from the NVSS catalog.

c Source identifications from SIMBAD. The identification codes are: A = AGN; B = BL Lac object; G = Galaxy; Q = Quasar.

${ }^{d}$ Spectral index $\alpha$ defined according to $S \sim \nu^{\alpha}$. The figure in parentheses represents the uncertainty in the last digit quoted in the measured value.

${ }^{\mathrm{e}}$ Radio survey used with the NVSS to determine the spectral index, where possible. The survey codes are: $7 \mathrm{C}=\mathrm{Seventh} \mathrm{Cambridge} \mathrm{Catalog}$ at $151 \mathrm{MHz}$ (Hales et al. 2007); TXS = The Texas Survey at $365 \mathrm{MHz}$ (Douglas et al. 1996); 87GB = 87 Green Bank Catalog at 4850 MHz (Gregory \& Condon 1991); PMN = Parkes-MIT-NRAO Survey at $4850 \mathrm{MHz}$ (Griffith \& Wright 1993).

${ }^{\mathrm{f}}$ Source not observed owing to telescope scheduling limitations.

TABLE 2

OBSERVING PARAMETERS FOR JODRELL BANK AND NRAO 43-M SURVEYS

\begin{tabular}{lcc}
\hline \hline \multicolumn{1}{c}{ Survey } & Crawford et al. (2000) & This Survey \\
\hline Number of Targets & 92 & 75 \\
Telescope & Lovell (Jodrell Bank) & NRAO \\
Telescope diameter $(\mathrm{m})$ & 76 & 43 \\
Central observing frequency, $\nu_{c}(\mathrm{MHz})$ & 610 & 1200 \\
Observing bandwidth (MHz) & 1 & 800 \\
Number of frequency channels & 32 & 4096 \\
Number of bits per sample & 1 & 8 \\
Integration time per source $(\mathrm{s})$ & 420 & 900 \\
Sampling time $(\mu \mathrm{s})$ & 50 & 61.44 \\
& & \\
\hline
\end{tabular}

